\title{
EXACTITUD ESPACIAL EN LA CREACIÓN DE BASES DE DATOS SIG MODELOS RÁSTER Y VECTORIAL
}

\section{SPATIAL ACCURACY IN THE CREATION OF DATABASES GIS RASTER AND VECTOR MODELS}

\author{
Carlos Mena Frau' ${ }^{1} \quad$ Yony Ormazábal Rojas² $^{2}$ Yohana Morales Hernández $^{3} \quad$ John Gajardo Valenzuela $^{4}$ \\ Recibido el 18 de julio de 2007, aceptado el 13 de noviembre de 2007 \\ Received: July 18, 2007 Accepted: November 13, 2007
}

\begin{abstract}
RESUMEN
La exactitud en la representación espacial de elementos geográficos en una base de datos de un Sistema de Información Geográfica (SIG) está sujeta a errores originados en los procesos de captura y procesamiento de los datos, lo cual queda en evidencia cuando se integra información más exacta. La cuantificación del error es fundamental cuando se pretende tomar decisiones sobre la base de la información disponible. En el estudio se establece una metodología para determinar y comparar el error de representación de elementos geográficos en los modelos vectorial y ráster. La base de datos incluye elementos puntuales, lineales y poligonales, que representan elementos reales correspondientes a una torre, un camino y un rodal. Esta información se obtuvo a partir de mediciones en terreno con el Sistema de Posicionamiento Global (GPS), que fueron procesadas para dar origen a coberturas en formato vectorial. Posteriormente, se realizó el traspaso de la información al formato ráster, empleando resoluciones de píxel de 5, 10 y 20 metros. Mediante un algoritmo matemático se determinaron coordenadas homólogas entre los elementos representados en los dos modelos. De la distancia euclidiana entre coordenadas homólogas, se obtuvo un error medio de representación para cada modelo que luego se utilizó para hacer un análisis estadístico de comparación de exactitud espacial entre los modelos. Los resultados obtenidos indican que no existen diferencias significativas en el error medio de los modelos vectorial y ráster, pero sí existen diferencias en los errores medios arrojados para las distintas resoluciones empleadas en la rasterización de la información original.
\end{abstract}

Palabras clave: Representación geográfica, exactitud espacial, modelo vectorial, modelo ráster, base de datos.

\begin{abstract}
Representation spatial accuracy of geographic elements in a Geographic Information System (GIS) database is subject to errors generated in the processes of data capture and processing, which is in evidence when more exact information is integrated. Error quantification is fundamental when the decision-making is based on the information available. The study establishes a methodology to determine and compare the representation error of geographic elements in the vector and raster models. Database includes point, line and polygon elements that represent real objects which are a tower, a road and a parcel. This information was obtained from land measurements with Global Positioning System (GPS) that were processed to obtain vector layers. Later, the convert of vectors layers into raster layers was made using pixel resolutions of 5, 10 and 20 meters. By means of a mathematical algorithm homologous coordinates between the elements represented in both models were determined. From the euclidean distance between homologous coordinates, a mean error of representation for each model was obtained that soon was used to make a statistical comparison analysis of space accuracy between models. The obtained results indicate that there are not significant differences between mean errors of vector and raster models, but there are differences between mean errors of the raster layers at different resolutions.
\end{abstract}

Keywords: Geographic representation, spatial accuracy, vector model, raster model, database.

\footnotetext{
1 Centro de Geomática. Universidad de Talca. Avenida Lircay s/n. Talca, Chile. E-mail: cmena@utalca.cl

2 Centro de Geomática. Universidad de Talca. Avenida Lircay s/n. Talca, Chile. E-mail: yormazabal@utalca.cl

3 Centro de Geomática. Universidad de Talca. Avenida Lircay s/n. Talca, Chile. E-mail: ymorales@utalca.cl

4 Centro de Geomática. Universidad de Talca. Avenida Lircay s/n. Talca, Chile. E-mail: jgajardo@utalca.cl
} 


\section{INTRODUCCIÓN}

La capacidad ofrecida por los Sistemas de Información Geográfica (SIG) de generar nueva información a partir de un conjunto de datos espaciales ha revolucionado el manejo y análisis de la información geográfica, convirtiéndose en una herramienta altamente especializada para la gestión de dicha información, lo cual se ve reflejado en la gran difusión de estos sistemas en diferentes sectores que manejan información espacial y temática asociada, principalmente para resolver problemas de tipo ambiental, social y económico.

La gran ventaja de gestionar la información espacialmente referenciada en formato digital ha significado una disminución en el tiempo de manipulación de ésta respecto a la utilización tradicional de soportes analógicos, acorde con la dinámica de los procesos de gestión del territorio en la actualidad, lo cual sitúa a los SIG como una herramienta de gran impacto en la gestión eficiente de los recursos por parte de las instituciones que disponen y utilizan dicha información.

La posibilidad de integrar una serie de datos espaciales heterogéneos es otra cualidad de los SIG, con lo cual se diversifica y amplía la capacidad de análisis sobre los recursos, derivando nueva y rica información que puede ser propagada y actualizada desde un set de datos a otro [1]. En este esquema, el proceso de entrada de datos al SIG tiene por finalidad generar una base de datos en formato digital correspondiente a una zona o área determinada del territorio [2]. De esta forma, una base de datos puede proceder de procesos concernientes a: topografía, fotogrametría, teledetección e informática [3].

En la actualidad, la demanda por la rápida disposición de bases de datos actualizadas está incrementándose, obligando a investigar, adaptar y evaluar nuevas técnicas y procedimientos de integración de información [4]. Elementos como modelos digitales de elevación (MDE), imágenes satelitales de distintos sensores, imágenes fotográficas aéreas digitales, imágenes líder o modelos 3D, cada día son de más fácil acceso, implicando un gran flujo de datos que debe ser integrado adecuadamente para obtener el máximo de provecho $[5,6]$.

Dentro de los modelos de datos empleados por los SIG es posible distinguir dos estructuras principales: vectorial y ráster [7]. El primero delinea los contornos de los elementos representados, por medio de vectores o polilíneas; mientras que el segundo divide el espacio en una matriz regular de celdillas o píxeles, en donde la representación de los elementos se realiza señalando la existencia o no de ellos dentro de cada píxel [8]. De esta forma, la representación de información geográfica, en uno u otro modelo, corresponde a una abstracción del territorio, en donde elementos geográficos complejos se representan por objetos geométricos simples: puntos, líneas y polígonos.

En este contexto, el error de representación de la información geográfica es un problema importante, debido a que frecuentemente los elementos representados presentan formas no regulares o vagas [9]. La magnitud de este error depende del proceso de captura de información espacial o del procesamiento aplicado, lo cual determinará la confiabilidad de la base de datos respecto a la verdadera ubicación de los elementos en el territorio. En este sentido, el término exactitud, indica el grado de conformidad de la información de la base de datos respecto de una norma establecida. Por su parte, el concepto de precisión indica la confiabilidad de dicha información.

En términos generales debiese existir una consistencia entre los objetos geométricos y los elementos geográficos del territorio, pero es posible detectar errores que pueden agruparse en tres categorías: los errores por fuentes obvias, como antigüedad de los datos, escalas, densidad de observaciones o área de cobertura; los errores resultantes de la variación natural de las medidas originales; y los errores surgidos durante la aplicación de los tratamientos utilizados. Los dos primeros generalmente son más fáciles de detectar, sin embargo, los últimos ofrecen una dificultad mayor en su identificación [10].

En este contexto, el objetivo de la presente investigación es generar coberturas con indicadores de exactitud espacial que permitan determinar la representatividad de la información y a la vez, comparar el error asociado a la representación de los modelos vectorial y ráster.

\section{CUERPO}

\section{Metodología}

La investigación tomó como objeto de estudio los elementos geográficos básicos que pueden ser representados en un SIG, éstos son: puntos, líneas y polígonos. Dichos elementos son graficados utilizando un sistema de coordenadas en el SIG vectorial, y un sistema de celdillas o píxeles en el SIG ráster. En la realidad, los elementos seleccionados y representados digitalmente correspondieron a una torre de vigilancia, un camino secundario de 1.300 metros de longitud y un rodal cubierto por bosque de aproximadamente 36 hectáreas. 


\section{Antecedentes de la zona de estudio}

Todos los elementos antes indicados se localizan en el cerro La Virgen ubicado en la zona central de la VII Región del Maule, específicamente en la Comuna de Talca. La zona presenta una altitud mínima de 110 m.s.n.m. y una máxima de 277 m.s.n.m. Su ubicación geográfica es $35^{\circ} 25^{\prime} 20^{\prime \prime}$ de latitud sur y $71^{\circ} 41^{\prime} 55^{\prime \prime}$ de longitud oeste. Para efectos del estudio, se empleó el Sistema de Proyección Universal Transversal de Mercator (UTM), el Elipsoide Internacional de 1924 y el datum Provisorio Sudamericano de 1956 (PSAD 56), Huso 19 Sur, como sistema de georreferenciación.

\section{Generación de bases de datos digitales}

Para tener elementos de comparación cuyo error de representación fuese conocido desde el comienzo del proceso, se optó por capturar la información en terreno por medio del Sistema de Posicionamiento Global (GPS). El equipo utilizado fue un GPS cartográfico (receptor móvil y base) marca Trimble Pro-XR de frecuencia simple y 12 canales. Dicho sistema ofrece la posibilidad de realizar mediciones cuyas precisiones, dependiendo de la modalidad y el tiempo de medición, pueden alcanzar unos pocos centímetros [11]. En el caso del elemento puntual torre, se utilizó el modo estático durante un lapso de 10 minutos. Para el elemento lineal camino, se realizaron mediciones en modo dinámico a intervalos de 5 segundos. Para el elemento poligonal rodal, también se utilizó el modo dinámico de medición a intervalos de 5 segundos. El tiempo de medición para estos dos últimos elementos varió dependiendo del tiempo de recorrido en cada caso. Posteriormente, el conjunto de datos recolectados en terreno fue sometido al proceso de corrección diferencial, cotejándolos con los datos del receptor GPS base. Dicho receptor base se localiza en el Centro de Geomática de la Universidad de Talca, con lo cual se obtuvieron líneas de base (distancia entre receptor móvil y base) de 5 kilómetros.

El resultado de esta primera etapa se exportó como archivos digitales a un SIG vectorial (ArcGIS), en donde se editaron y corrigieron los errores gráficos para luego asignar el sistema de georreferenciación indicado anteriormente. De esta forma, se obtuvo una base de datos vectorial compuesta por tres capas de información: puntos, líneas y polígonos.

En la etapa posterior se procedió a exportar dichas capas de información individualmente hasta un SIG ráster (IDRISI) en donde se procedió a rasterizar cada una de ellas. Aunque el software ArcGIS posee la capacidad de trabajar directamente con archivos ráster, se optó por el uso del software IDRISI en esta fase debido a las mayores funcionalidades que éste ofrece en el tratamiento y manejo de información en formato ráster. La rasterización involucra la conversión de la información de formato digital vectorial a formato digital ráster, mediante un procedimiento de presencia o ausencia de objetos geográficos en los píxeles de una matriz de igual extensión geográfica que la cobertura vectorial original [12]. A modo de evaluar el efecto del error de representación ráster asociado a la resolución espacial de los píxeles definidos para la matriz, se generaron tres niveles de resolución correspondientes a 5,10 y 20 metros para la representación de cada elemento geográfico considerado en el estudio.

\section{Determinación de error de representación vectorial y ráster}

El error de representación digital vectorial de los elementos punto, línea y polígono se determinó a partir de las mediciones realizadas con GPS. Para esto, se utilizó una serie de posiciones medidas en terreno para cada elemento, cuyas precisiones fueron obtenidas desde la corrección diferencial efectuada. Para cada set de datos, se procedió a calcular el error medio y la desviación estándar de exactitud espacial. Según el autor Felicísimo [13] el error puntual se puede evaluar mediante el Error Cuadrático Medio (ECM) o Error Medio (EM). De esta forma, se obtuvo un error asociado a cada elemento de la base de datos, el cual se estableció como base de comparación para determinar el error de posicionamiento en la representación ráster de los mismos.

De acuerdo al autor Calvo [3], para cuantificar el error de representación ráster se puede comparar los datos de posición de un elemento presente en la cobertura ráster con otros datos del mismo elemento representado en otro modelo que presente una mayor exactitud espacial, mediante sus coordenadas homólogas. De este modo, se tendrán mediciones de la diferencia entre estas coordenadas, de las cuales es posible obtener una media de las diferencias o Error Medio (EM) y una desviación estándar, que permita realizar una prueba de calidad para el modelo al comparar el EM obtenido, con un valor máximo aceptable, mediante la siguiente expresión:

$$
T z=\frac{(|\bar{\delta}|-\delta z o) * \sqrt{n}}{\mathrm{~S} \delta}
$$

donde:

$\mathrm{T} z=$ prueba $\mathrm{T}$ de Student

$|\bar{\delta}|=$ valor medio de las diferencias.

$\delta z o=$ error máximo aceptable.

$n=$ número de observaciones.

$\mathrm{S} \delta=$ desviación estándar de las diferencias. 


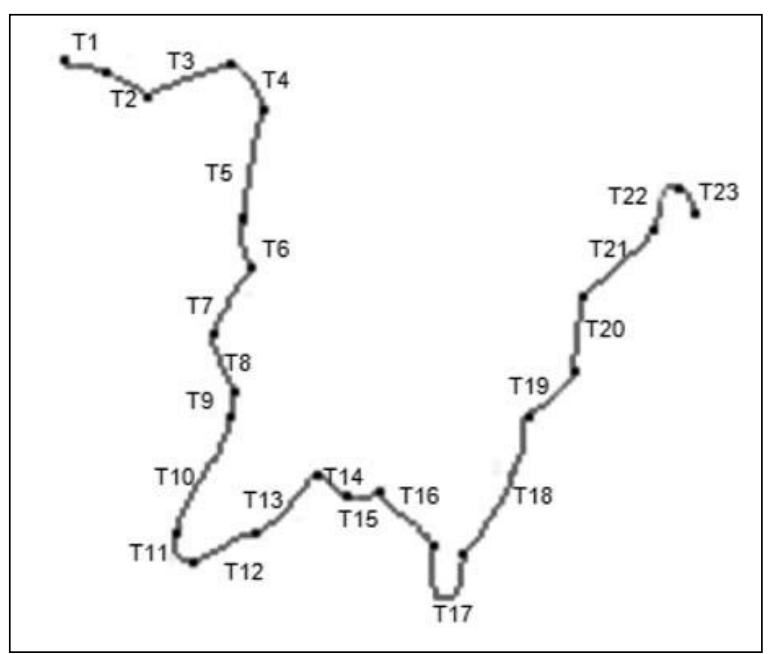

Figura 1. Representación espacial del elemento lineal (modelo vectorial).

Los autores Ariza, López, López-Luque y Pinilla [14] señalan que el error máximo aceptable para una representación ráster corresponde a la semidiagonal del píxel empleado. Este valor se puede determinar por medio de la ecuación:

$$
E m=\frac{T}{\sqrt{2}}
$$

donde T corresponde a la resolución espacial del píxel.

De acuerdo a lo señalado anteriormente, las coberturas ráster a evaluar presentan una resolución de 5, 10 y 20 metros, por lo que los errores máximos admisibles serán $3,54 \mathrm{~m}, 7,07 \mathrm{~m}$ y $14,14 \mathrm{~m}$, respectivamente.

El error de representación de cada elemento geográfico a resoluciones de 5, 10 y 20 metros se obtuvo usando la siguiente metodología:

- Para el punto, el error ráster corresponde a la distancia euclidiana entre el centro del píxel y la coordenada vectorial obtenida con GPS.

- Para determinar el error de la línea y el polígono previamente se definió una serie de tramos en el modelo vectorial, de manera de facilitar el proceso

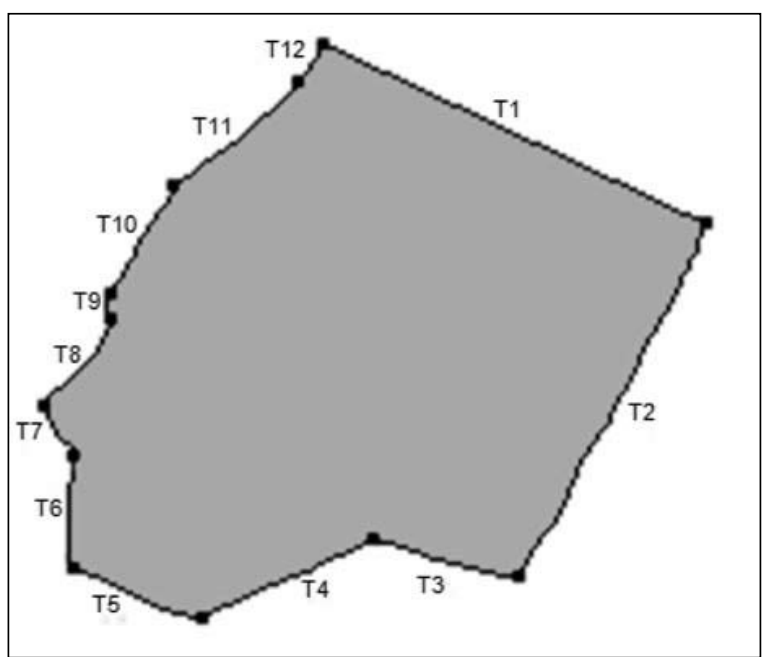

Figura 2. Representación espacial del elemento poligonal (modelo vectorial).

(ver figura 1 y figura 2). Luego se superpuso la cobertura vectorial y la cobertura ráster. De esta última se obtuvieron puntos de control para cada tramo, correspondientes al centro de los píxeles que definen dichos tramos (la ubicación geográfica de cada píxel está georreferenciada en su centro [15]). Luego, aplicando el algoritmo de determinación de coordenadas homólogas, se identificaron las coordenadas homólogas sobre la línea vectorial. De esta forma, se determinó el error de representación usando la distancia euclidiana entre coordenadas homólogas.

\section{Algoritmo de determinación de coordenadas homólogas entre bases de datos}

En la figura 3 se identifican dos pares de coordenadas que en teoría corresponden a coordenadas homólogas de la fuente de datos original (en este caso la cobertura ráster) y la base de datos final (cobertura vectorial).

La metodología que se utilizó para obtener coordenadas homólogas entre bases de datos espaciales, es la siguiente: en primer lugar, se definió un par de coordenadas (p1,p2) (ver figura 3) que se ubican sobre la línea de la fuente original (cobertura ráster); falta entonces conocer el par homólogo (x,y) que se ubica en la línea de la base de datos final (cobertura vectorial). 


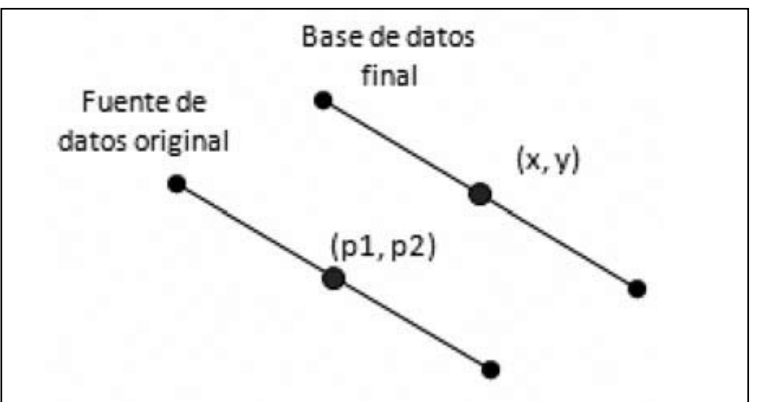

Figura 3. Coordenadas homólogas entre Bases de Datos Espaciales.

En la figura 4 se denotan las principales coordenadas necesarias para obtener el par de coordenadas homólogas. Se definió a los pares $(a, b)$ y $(c, d)$ como las coordenadas de los extremos de la línea vectorial, denotada como vector $A$. Por lo tanto, este vector tendrá como componentes $(c-a, d-b)$.

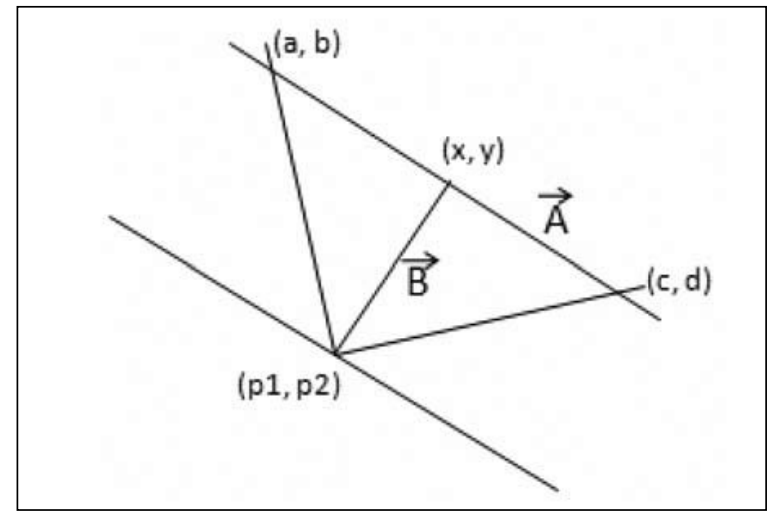

Figura 4. Identificación de principales coordenadas de Bases de Datos.

En la figura 3 se denotó como $p 1$ y p2 a las coordenadas vectoriales de la fuente de datos original (cobertura ráster) $(p 1=a$ y $p 2=d) ; x$ e $y$, a la coordenada vectorial homóloga sobre el vector $A$ (cobertura vectorial).

Con estos datos de coordenadas geográficas, se generó un nuevo vector (vector $B)$, cuyos componentes son: $(x-p 1, y-p 2)$, y que es perpendicular al vector $A$. De esta información se desprende el siguiente sistema de ecuaciones de la pendiente de la línea vectorial y el producto punto entre los vectores $A$ y $B$, para obtener las coordenadas $(x, y)$ homólogas:

\section{-Ecuación a partir de la pendiente de la línea A:}

$$
m=(b-d) /(a-c)=m 1
$$

donde $m l$ es un valor conocido que puede expresarse en función de $x$ e $y$ :

$$
m l=(d-y) /(c-x) \Rightarrow y=-m l(c-x)+d
$$

\section{-Ecuación definida del producto punto entre los} vectores A y B:

Los vectores $A$ y $B$ tienen los siguientes componentes: $\vec{A}=(c-a, d-b), \vec{B}=(x-p 1, y-p 2)$. Estos vectores al ser perpendiculares $(\vec{A} \perp \vec{B})$, su producto punto es cero [16], entonces:

$$
\vec{A} \cdot \vec{B}=0 \Rightarrow(c-a, d-b) \cdot(x-p 1, y-p 2)=0
$$

Desarrollando el producto punto:

$$
(c-a)(x-p 1)+(d-b)(y-p 2)=0
$$

Debido a que la diferencia $c-a$ y $d-b$ son constantes, se denotó por $\theta$ y $\alpha$ respectivamente, para simplificar la ecuación. Reemplazando la ecuación 1 en la ecuación 2 : $\theta(x-p 1)+\alpha((d-m 1(c-x))-p 2)=0$

Entonces, se desarrolla esta ecuación para obtener el valor de $x$ :

$$
x=(-\theta p 1+\alpha d-\alpha m 1 c-\alpha p 2) /(-\theta-\alpha m 1)
$$

Reemplazando $\theta$ y $\alpha$ en la ecuación:

$$
x=(-(c-a) p 1+(d-b) d-(d-b) m l c-(d-b) p 2) /(-(c-a)-(d-b) m l)
$$

Pero, $p 2=d$; entonces, el valor de $x$ sobre la línea vectorial A es:

$$
x=(-(c-a) p 1-(d-b) m 1 c) /(-(c-a)-(d-b) m 1)=x^{\prime}
$$

Por lo tanto, reemplazando $x$ en la ecuación 1 , el valor de $y$ es:

$$
y=d-m 1\left(c-x^{\prime}\right)
$$

Al desarrollar estas ecuaciones, finalmente se tendrán pares de coordenadas $(p 1, p 2)$ de la fuente original y su par homóloga $(x, y)$, sobre la línea vectorial final, para luego determinar el error de representación mediante la distancia euclidiana entre estas coordenadas.

\section{Comparación de la exactitud de representación}

Con el fin de comparar la exactitud espacial del modelo ráster, respecto de la representación vectorial, se aplicó la distribución $t$ de Student, en cada nivel de resolución y de acuerdo al máximo error aceptable definido previamente. 
Por otra parte, para determinar si existen diferencias significativas entre el error medio obtenido entre los niveles de resolución 5, 10 y 20 metros de píxel, se realizó un análisis de varianza considerando todos los datos de error medio para los trazados pertenecientes a cada elemento (variable independiente) y los niveles de resolución (variable dependiente).

\section{RESULTADOS}

\section{Representación de elementos geográficos en formato digital vectorial}

Luego de los análisis correspondientes, se determinó que el error medio de representación de la base de datos digital vectorial obtenida con GPS, corresponde a:

- $\quad$ 0,15 metros para el elemento puntual torre, que se define como un radio alrededor de la coordenada centro, con una desviación estándar de 0,06 metros.

- 1,0 metros para el elemento lineal camino. En este caso la exactitud geográfica se obtuvo del error medio de un grupo de mediciones o posiciones capturadas con GPS, que generan un corredor a cada lado de la línea capturada; la variabilidad de los datos, medida mediante la desviación estándar, es de 0,4 metros.

- 1,6 metros para el elemento poligonal rodal con una desviación estándar de 0,7 metros (en los tres casos, la desviación estándar incluye el error asociado al equipo GPS utilizado en las mediciones).

\section{Representación de elementos geográficos en formato digital ráster}

Como se indicó anteriormente, debido a la irregularidad de las líneas que presentan los elementos lineal y poligonal, se dividió cada elemento en tramos (ver figura 1 y figura 2), de manera de aplicar el algoritmo de determinación de coordenadas homólogas y tener un mejor control de comparación entre los niveles de resolución.

a) Representación del elemento puntual: en la torre de vigilancia se determinó el error de representación ráster mediante el cálculo de la distancia euclidiana entre las coordenadas $(\mathrm{X}, \mathrm{Y})$ vectoriales, obtenidas en terreno con GPS $(255.389,6$ E y 6.078.193,0 N) y la coordenada de vinculación vectorial del centro del píxel que representa a la torre en el modelo ráster.

- Para la resolución 5 metros, la coordenada de vinculación corresponde a 255.387,5 E y 6.078.193,0 $\mathrm{N}$; por lo tanto, el error de exactitud espacial es de 2,1 metros (ver figura 5).

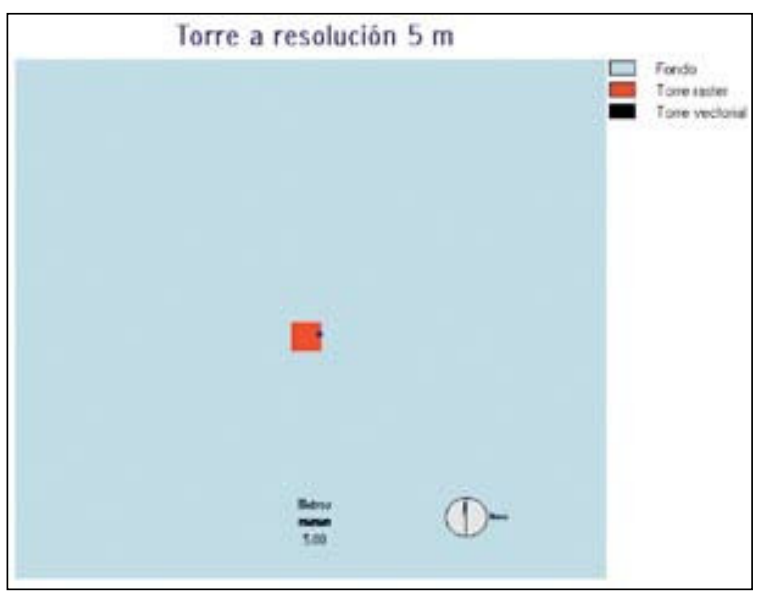

Figura 5. Representación (ráster y vectorial) del elemento puntual a resolución de 5 metros.

- A resolución de 10 metros, presenta las coordenadas de vinculación 255.385,0 E y 6.078.195,0 N y un error de representación de 5 metros (ver figura 6).

- A nivel de resolución 20 metros, presenta las coordenadas centrales del píxel a $255.390,0 \mathrm{E}$ y 6.078.190,0 $\mathrm{N}$ y un error de 3 metros (ver figura 7).

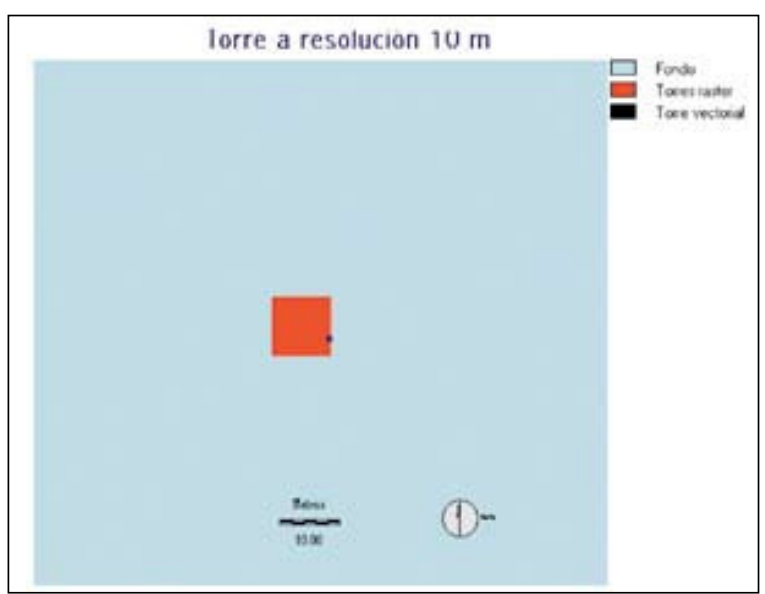

Figura 6. Representación (ráster y vectorial) del elemento puntual a resolución de 10 metros.

Finalmente, considerando el error determinado para la medición de los datos con GPS, el error total de representación del elemento puntual en el modelo ráster es de 2,25 metros a resolución 5 metros; 5,15 metros a resolución 10 metros; y 3,15 metros al nivel de resolución 20 metros. 


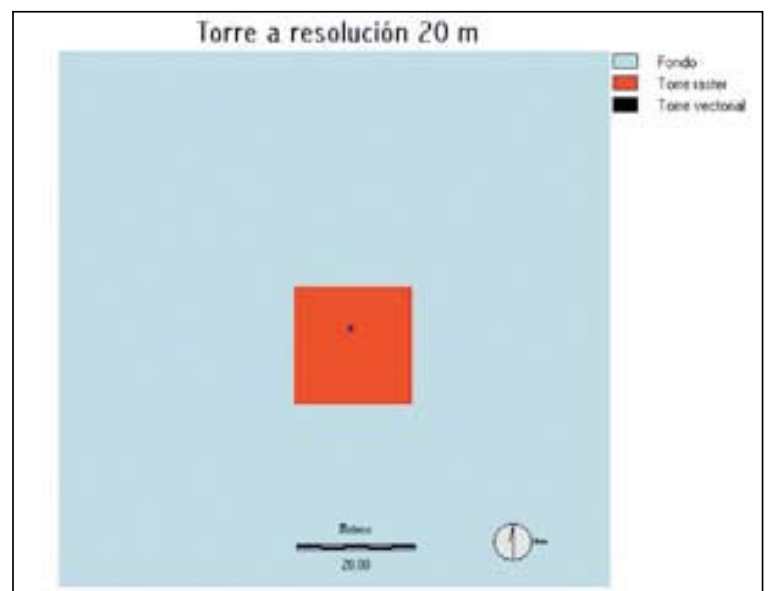

Figura 7. Representación (ráster y vectorial) del elemento puntual a resolución de 20 metros.

b) Representación del elemento lineal: la tabla 1 presenta el error medio (EM) y la desviación estándar asociado a los niveles de resolución de 5, 10 y 20 metros de píxel para la representación del camino en el modelo ráster, el cual se dividió en 23 tramos (ver figura 1). En las figuras 8,9 y 10 se puede apreciar la representación del elemento lineal camino en el modelo ráster a resolución de 5, 10 y 20 metros, respectivamente.

Si se suma el error de medición GPS a los errores medios totales de la tabla 1, tenemos que el error medio final es de 2,91 para la resolución de 5 metros; 4,18 para la resolución de 10 metros y 7,14 para la resolución de 20 metros. El resultado obtenido del análisis de varianza estableció como $\mathrm{H}_{0}$ la igualdad entre errores medios totales obtenidos para cada resolución, mientras que la hipótesis nula establecía diferencias entre dichos errores. A un nivel de significación de 0,01 (99\% de confianza) con 2 grados de libertad para las resoluciones $(5,10$ y $20 \mathrm{~m})$ y 66 grados de libertad entre grupos (23 tramos por resolución), se obtuvo un valor de tabla $\mathrm{F}_{0,01(2,66)}=4,95$; mientras que el F calculado fue de 62,81. De esta forma, como el F calculado es mayor al F de tabla 1, se rechaza la hipótesis nula, indicando que el error medio entre los niveles de resolución es estadísticamente significativo.

En cuanto a la prueba $t$ de Student, no se aplicó en este caso debido a que los errores arrojados para las resoluciones 5, 10 y 20 metros $(2,91 ; 4,18$ y 7,14; respectivamente) fueron menores a los errores máximos admisibles para tales resoluciones (3,54; 7,07 y 14,14; respectivamente), con lo cual dichos errores de representación en el modelo ráster están dentro del corredor de error permitido, indicando una buena exactitud en la representación de los elementos.
Tabla 1. Error espacial del camino en el modelo ráster a resolución 5, 10 y $20 \mathrm{~m}$.

\begin{tabular}{|c|c|c|c|c|c|c|}
\hline \multirow{2}{*}{\begin{tabular}{|c|}
$\mathbf{T}$ \\
$\mathbf{R}$ \\
$\mathbf{A}$ \\
$\mathbf{M}$ \\
$\mathbf{O}$ \\
$\mathbf{S}$
\end{tabular}} & \multicolumn{2}{|c|}{ Resolución $5 \mathrm{~m}$} & \multicolumn{2}{|c|}{ Resolución $10 \mathrm{~m}$} & \multicolumn{2}{|c|}{ Resolución $20 \mathrm{~m}$} \\
\hline & $\begin{array}{l}\text { Error } \\
\text { Medio } \\
\text { (m) }\end{array}$ & $\begin{array}{l}\text { Desv. } \\
\text { Estándar } \\
\text { (m) }\end{array}$ & $\begin{array}{c}\text { Error } \\
\text { Medio } \\
(\mathrm{m})\end{array}$ & $\begin{array}{c}\text { Desv. } \\
\text { Estándar } \\
\text { (m) }\end{array}$ & $\begin{array}{c}\text { Error } \\
\text { Medio } \\
(\mathrm{m})\end{array}$ & $\begin{array}{l}\text { Desv. } \\
\text { Estándar } \\
\text { (m) }\end{array}$ \\
\hline $\mathrm{T} 1$ & 1,51 & 0,87 & 1,42 & 1,12 & 6,81 & 2,25 \\
\hline $\mathrm{T} 2$ & 1,53 & 1,27 & 3,19 & 1,94 & 6,87 & 4,84 \\
\hline $\mathrm{T} 3$ & 2,44 & 1,68 & 3,66 & 2,09 & 7,86 & 4,92 \\
\hline $\mathrm{T} 4$ & 2,57 & 1,55 & 3,59 & 2,06 & 8,92 & 5,05 \\
\hline T5 & 1,91 & 1,35 & 2,71 & 1,64 & 4,02 & 3,08 \\
\hline T6 & 1,74 & 0,98 & 2,85 & 1,69 & 2,76 & 3,11 \\
\hline $\mathrm{T} 7$ & 1,70 & 1,70 & 3,58 & 2,68 & 6,34 & 5,03 \\
\hline T8 & 1,52 & 1,04 & 2,59 & 2,15 & 4,38 & 5,14 \\
\hline T9 & 1,74 & 0,69 & 0,99 & 0,66 & 6,31 & 0,94 \\
\hline T10 & 1,90 & 1,51 & 3,41 & 2,25 & 5,78 & 4,40 \\
\hline T11 & 1,58 & 1,02 & 3,70 & 2,03 & 10,56 & 1,58 \\
\hline $\mathrm{T} 12$ & 2,42 & 1,51 & 2,94 & 2,23 & 3,96 & 0,28 \\
\hline T13 & 2,27 & 2,22 & 3,25 & 3,43 & 7,22 & 7,80 \\
\hline T14 & 2,09 & 1,49 & 3,88 & 2,58 & 6,62 & 3,34 \\
\hline $\mathrm{T} 15$ & 1,76 & 1,42 & 2,46 & 1,04 & 3,03 & 1,48 \\
\hline T16 & 3,58 & 1,70 & 4,00 & 3,78 & 3,89 & 3,31 \\
\hline T17 & 1,12 & 1,28 & 2,81 & 0,50 & 6,08 & 3,30 \\
\hline T18 & 2,11 & 1,69 & 3,12 & 2,22 & 6,71 & 3,00 \\
\hline T19 & 2,32 & 1,67 & 3,38 & 1,80 & 6,76 & 6,10 \\
\hline T20 & 2,10 & 1,24 & 2,92 & 2,23 & 2,82 & 2,09 \\
\hline $\mathrm{T} 21$ & 1,37 & 1,41 & 3,47 & 0,79 & 6,95 & 3,17 \\
\hline T22 & 1,26 & 0,95 & 2,19 & 2,02 & 5,61 & 3,11 \\
\hline $\mathrm{T} 23$ & 1,14 & 0,77 & 4,07 & 0,89 & 5,52 & 3,78 \\
\hline TOTAL & 1,91 & 1,46 & 3,18 & 2,09 & 6,14 & 3,93 \\
\hline
\end{tabular}

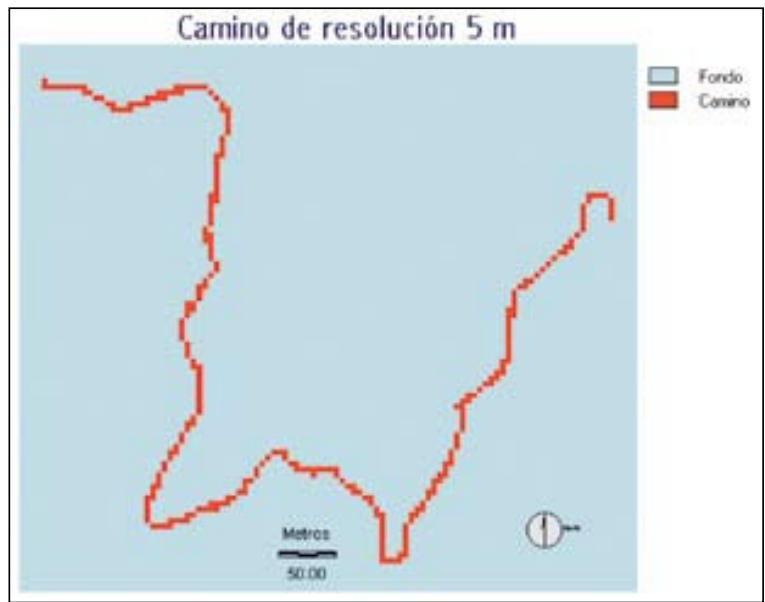

Figura 8. Representación ráster del elemento lineal a resolución de 5 metros. 


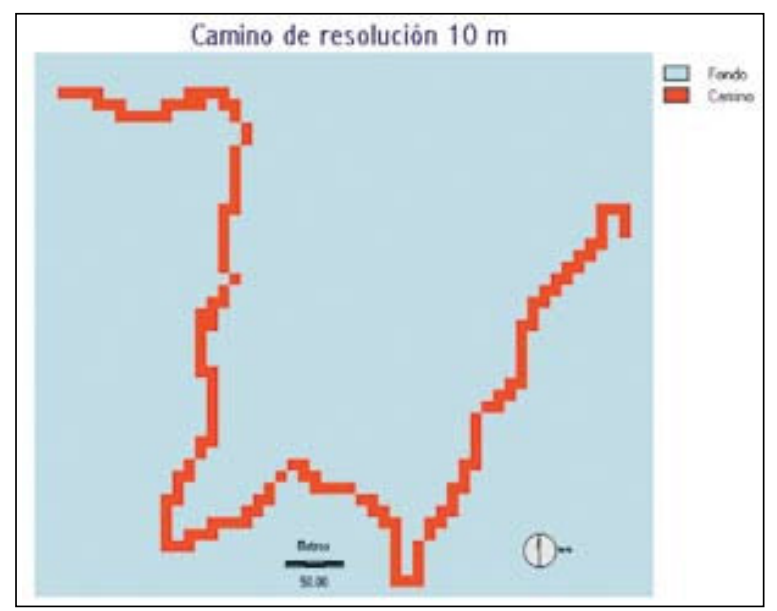

Figura 9. Representación ráster del elemento lineal a resolución de 10 metros.

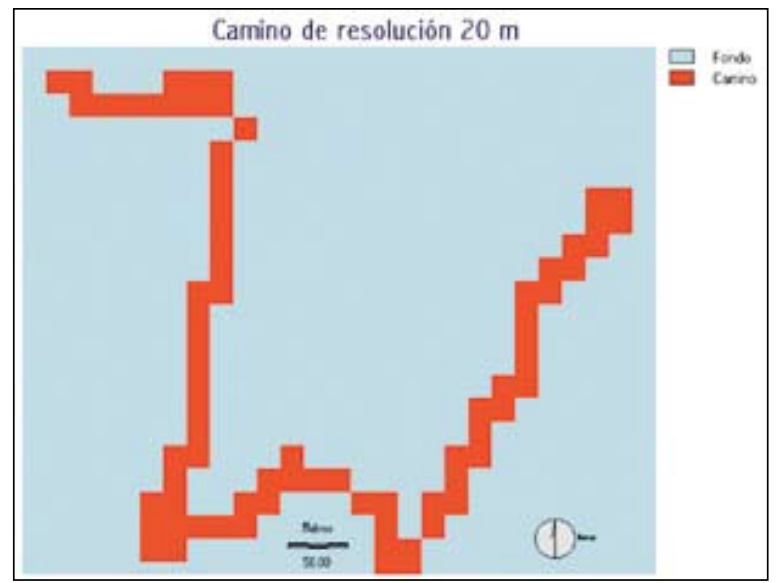

Figura 10. Representación ráster del elemento lineal a resolución de 20 metros.

c) Representación del elemento poligonal: en la tabla 2 se presenta el EM y la desviación estándar a niveles de resolución de 5, 10 y 20 metros de píxel para la representación del rodal en el modelo ráster, el cual se dividió en 12 tramos (ver figura 2).

Sumando el error estimado para la medición GPS de terreno, se establece que el error medio final de representación del rodal en el modelo ráster es de 5,13 para la resolución de 5 metros; 8,8 para la resolución 10 metros y 18,1 para la resolución 20 metros. En las figuras 11, 12 y 13 se puede observar una representación del elemento poligonal rodal en el modelo ráster a las distintas resoluciones.
Tabla 2. Error espacial del rodal en el modelo ráster a resolución 5,10 y 20 m.

\begin{tabular}{|c|c|c|c|c|c|c|}
\hline \multirow{2}{*}{$\begin{array}{c}\text { R } \\
\mathbf{A}\end{array}$} & \multicolumn{2}{|c|}{ Resolución 5 m } & \multicolumn{2}{|c|}{ Resolución 10 m } & \multicolumn{2}{|c|}{$\begin{array}{c}\text { Resolución } \\
\mathbf{2 0} \mathbf{~ m}\end{array}$} \\
\cline { 2 - 8 } $\begin{array}{c}\mathbf{M} \\
\mathbf{O} \\
\mathbf{S}\end{array}$ & $\begin{array}{c}\text { Error } \\
\text { Medio } \\
(\mathbf{m})\end{array}$ & $\begin{array}{c}\text { Desv. } \\
\text { Estándar } \\
(\mathbf{m})\end{array}$ & $\begin{array}{c}\text { Error } \\
\text { Medio } \\
(\mathbf{m})\end{array}$ & $\begin{array}{c}\text { Desv. } \\
\text { Estándar } \\
(\mathbf{m})\end{array}$ & $\begin{array}{c}\text { Error } \\
\text { Medio } \\
(\mathbf{m})\end{array}$ & $\begin{array}{c}\text { Desv. } \\
\text { Estándar } \\
(\mathbf{m})\end{array}$ \\
\hline T1 & 3,28 & 2,31 & 7,19 & 4,62 & 16,79 & 8,29 \\
\hline T2 & 3,68 & 2,38 & 7,06 & 4,72 & 18,15 & 7,23 \\
\hline T3 & 3,71 & 2,53 & 6,74 & 4,62 & 17,33 & 4,63 \\
\hline T4 & 3,78 & 2,47 & 7,06 & 4,57 & 16,78 & 9,14 \\
\hline T5 & 4,11 & 2,78 & 6,72 & 4,79 & 13,70 & 8,76 \\
\hline T6 & 3,16 & 1,77 & 8,28 & 2,51 & 17,59 & 8,11 \\
\hline T7 & 3,82 & 2,62 & 8,82 & 3,81 & 15,28 & 8,08 \\
\hline T8 & 3,26 & 2,45 & 6,88 & 4,85 & 18,37 & 8,54 \\
\hline T9 & 3,16 & 2,41 & 8,34 & 6,41 & 16,85 & 8,96 \\
\hline T10 & 3,12 & 2,41 & 6,99 & 4,84 & 15,55 & 9,51 \\
\hline T11 & 3,52 & 2,10 & 7,53 & 3,90 & 14,73 & 7,06 \\
\hline T12 & 3,35 & 2,36 & 7,43 & 5,12 & 13,34 & 6,22 \\
\hline T0TAL & $\mathbf{3 , 5 3}$ & $\mathbf{2 , 3 5}$ & $\mathbf{7 , 2 0}$ & $\mathbf{4 , 4 7}$ & $\mathbf{1 6 , 5 0}$ & $\mathbf{7 , 6 5}$ \\
\hline
\end{tabular}

En cuanto al análisis de varianza, se estableció como $\mathrm{H}_{0}$ la igualdad entre errores medios totales obtenidos para cada resolución, mientras que la hipótesis nula implica diferencias entre ellos. Para un nivel de significación igual a 0,01 (99\% de confianza) con 2 grados de libertad para las resoluciones $(5,10$ y $20 \mathrm{~m})$ y 33 grados de libertad entre grupos (12 tramos por resolución), se determinó un $\mathrm{F}_{0,01(2,33)}=5,33$; el $\mathrm{F}$ calculado se estimó en 451,81. De esta forma, como el $\mathrm{F}$ calculado es mayor al $\mathrm{F}$ de tabla 2, se rechaza la hipótesis nula, estableciendo que existen diferencias significativas del error medio entre los niveles de resolución.

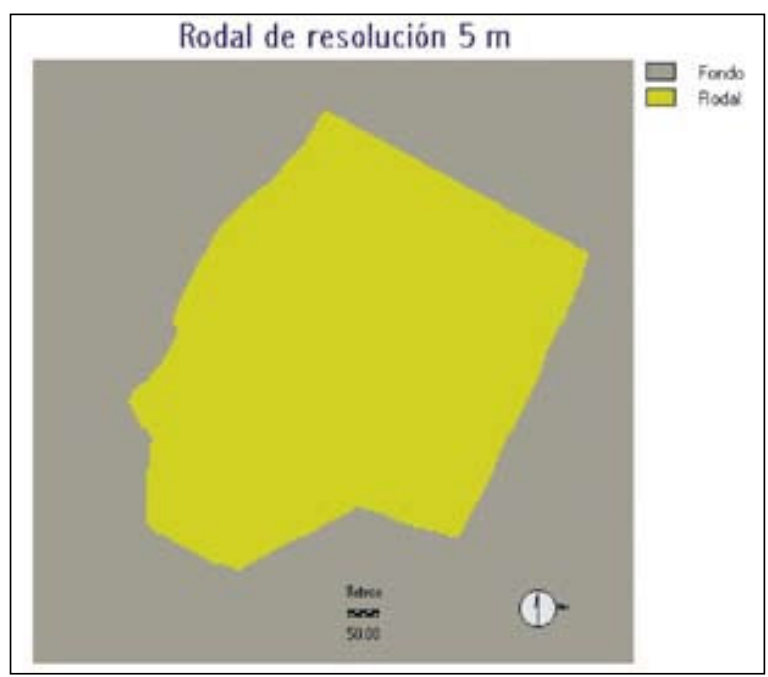

Figura 11. Representación ráster del elemento poligonal a resolución de 5 metros. 


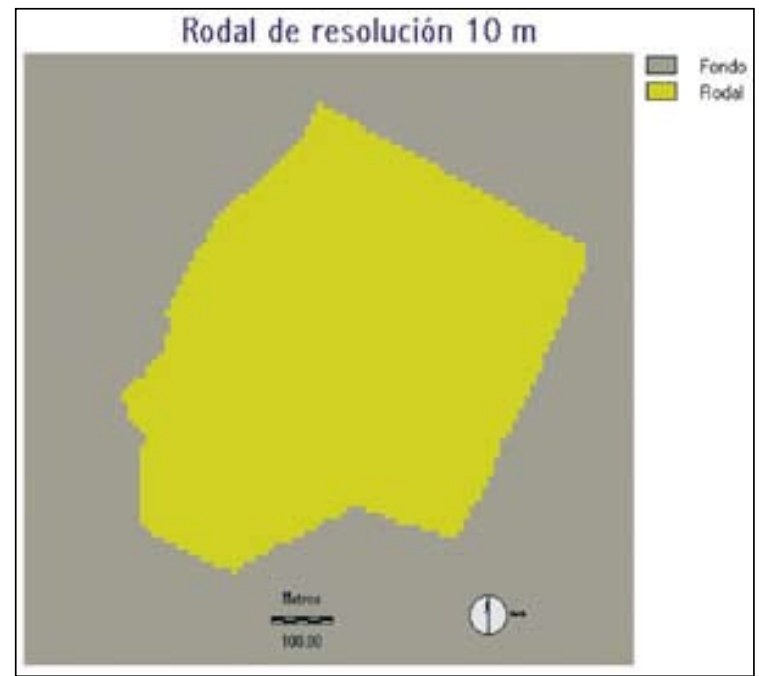

Figura 12. Representación ráster del elemento poligonal a resolución de 10 metros.

Para la prueba $t$ de Student, se estableció una $\mathrm{H}_{0}$ indicando igualdad de errores medios entre la representación vectorial y ráster (a distintas resoluciones), mientras que la hipótesis nula indicaba diferencia entre los mismos. Para un nivel de significación de 0,01 (99\% de confianza) con 11 grados de libertad, se tiene que $\mathrm{t}_{(11) 0,01}$ es igual a 2,718. En tanto, los $\mathrm{T}$ calculados para niveles de resolución de 5, 10 y 20 metros, considerando un error máximo admisible de 3,$54 ; 7,07$ y 14,14 ; respectivamente, correspondieron a 2,$342 ; 1,338$ y 1,792 . De esta forma, en todos los casos el t calculado es menor que el t de tabla, por lo que se acepta la $\mathrm{H}_{0}$, indicando que no existen diferencias significativas entre la representación del rodal en los modelos ráster y vectorial.

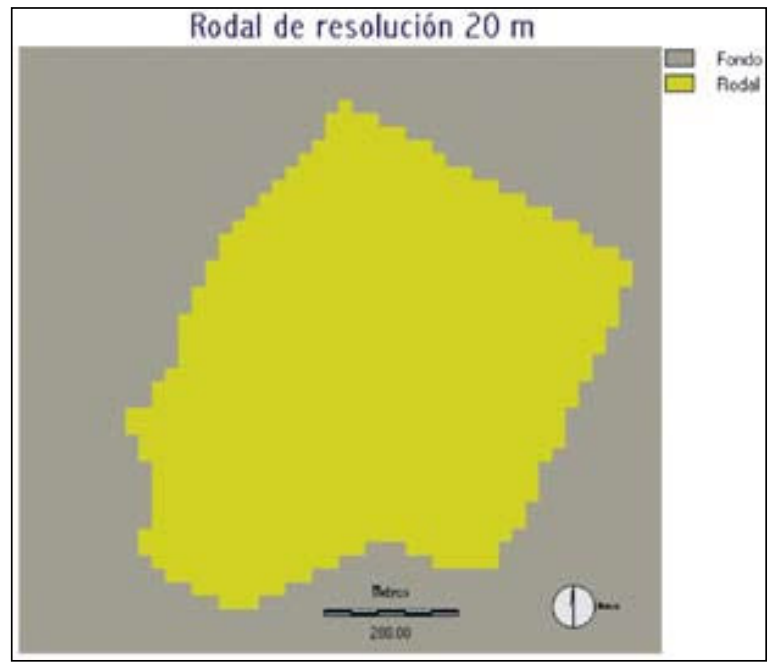

Figura 13. Representación ráster del elemento poligonal a resolución de 20 metros.

\section{CONCLUSIONES}

- La captura de información geográfica mediante el uso de tecnología GPS es más exacta que los métodos tradicionales. Dependiendo del método de medición, es posible alcanzar precisiones centimétricas, dando con ello una alta confiabilidad en la exactitud de la información generada a partir de datos medidos con receptores GPS y luego representada en una Base de Datos geográfica digital. Elementos puntuales, lineales y poligonales representados vectorialmente a partir de datos GPS ofrecen una alta exactitud espacial debido a que son generados a través de un proceso de captura de información directa en terreno. En el caso de la rasterización, el error se incrementa debido a la suma del error asociado a la fuente original (sea analógica o digital) y el error asociado al proceso de traspaso de dicha información. Esto sucede ya que el modelo ráster presenta un error espacial propio de su aplicación que debe sumarse al error que presenta la fuente original, para obtener el error total de representación.

- La resolución del píxel al momento de realizar una rasterización de información es un elemento clave en el resultado final de la exactitud obtenida en la representación de los distintos elementos geográficos. De esta forma, se estableció que para elementos lineales y poligonales, el error medio aumenta al aumentar el tamaño del píxel, registrándose diferencias estadísticas significativas entre las distintas resoluciones estudiadas. No obstante, dichos errores se encuentran bajo el límite máximo permitido, por lo que la representación de dichos elementos en los diferentes niveles de resolución, sigue siendo confiable.

- En el caso del elemento puntual, se registró que el error de representación en el modelo ráster no necesariamente es proporcional al tamaño de píxel, es decir que a mayor resolución no se obtiene un error espacial proporcionalmente mayor, ya que la magnitud del mismo va a depender de la ubicación del elemento puntual en la fuente original respecto al centro del píxel que lo representa, además de las coordenadas de origen de la matriz. En este sentido, se recomienda replicar la investigación considerando un mayor número de puntos de manera de realizar análisis más detallados.

- El elemento poligonal presenta mayor error de representación espacial que el elemento lineal en el modelo ráster, lo cual se encuentra asociado a su vez a un mayor error en las mediciones GPS de terreno para el polígono. Este mayor error se debe a que las 
características del elemento y las condiciones en las que se realizaron las mediciones en terreno (rodal cubierto con bosque adulto) disminuyeron la calidad de recepción de la señal GPS y, por ende, las precisiones finales obtenidas. Si bien los errores de medición GPS del polígono y la línea no muestran diferencias demasiado amplias entre sí, su efecto al momento de rasterizar dichos elementos puede determinar una diferencia mayor en las representaciones digitales finales de los mismos.

- Finalmente, es importante señalar que la exactitud de representación espacial de elementos geográficos dependerá directamente del proceso de captura usado para obtener dicha información. Por esto, es indispensable conocer los errores generados en la creación de bases de datos, de manera de establecer la real representatividad de la información que esta contiene y con ello asegurar un correcto uso y resultados satisfactorios de los procesos de análisis que tomen dicha información como base de decisión.

\section{REFERENCIAS}

[1] M. Butenuth, G. Gösseln, M. Tiedge, Ch. Heipke, U. Lipeck and M. Sester. "Integration of heterogeneous geospatial data in a federated database". ISPRS Journal of Photogrammetry and Remote Sensing. The Netherlands. In Press, Corrected Proof. Date of visit: 1 June 2007.

[2] J. Bosque. "Sistemas de Información Geográfica". Rialp S.A. Segunda Edición, p. 439. Madrid, España. 1997.

[3] M. Calvo. "Sistemas de Información Geográfica Digitales. Sistemas Geomáticos". Vitoria: Instituto Vasco de Administración Pública y Fundación EUSKOIKER, p. 616. España. 1993.

[4] C. Armenakis, F. Leduc, I. Cyr, F. Savopol and F. Cavayas. "A comparative analysis of scanned maps and imagery for mapping applications". ISPRS Journal of Photogrammetry \& Remote Sensing. The Netherlands. Vol. 57, pp. 304-314. 2003.

[5] U. Soergel, K. Schulz, U. Thoennessen and U. Stilla. "Integration of 3D data in SAR mission planning and image interpretation in urban areas". Information Fusion. USA. Vol. 6 N $^{\circ}$ 4, pp. 301-310. 2005.
[6] B. Tellez and S. Servigne. "Updating urban database with aerial photographs: A common structuring methodology". Computers, Environment and Urban Systems. UK. Vol. 21 No $^{\circ}$, pp. 133-145. 1997.

[7] C. Mena. "Geomática para la ordenación del territorio". Talca: Editorial Universidad de Talca. Chile, p. 316. 2005.

[8] Y. Lee, Z. Li and Y. Li. "Taxonomy of space tessellation". ISPRS Journal of Photogrammetry \& Remote Sensing. The Netherlands. Vol. 55, pp. 139-149. 2000.

[9] M. Schneider. "Design and implementation of finite resolution crisp and fuzzy spatial objects". Data \& Knowledge Engineering. USA. Vol. 44 $\mathrm{N}^{\mathrm{o}}$ 1, pp. 81-108. 2003.

[10] P. Burrough and R. McDonnell. "Principles of Geographical Information Systems". Oxford University Press. New York, USA, pp. 333. 1998.

[11] Trimble. "Sistemas de procesamiento de datos GPS compatibles con Sistemas de Información Geográfica”. Documento técnico de capacitación de INCOM S.A., p. 41. Santiago, Chile. 1999.

[12] J. Gutiérrez and M. Gould. "SIG: Sistemas de Información Geográfica”. Síntesis, p. 251. Madrid, España. 1994.

[13] A. Felicísimo. "Modelos Digitales del Terreno. Introducción y Aplicaciones en las Ciencias Ambientales". Pentalfa, p. 220. Oviedo, España. 1994.

[14] F. Ariza, A. López, R. López-Luque y C. Pinilla. "Uso de la simulación en el control de errores en la rasterización”. Revista Mapping. Madrid. Vol. 39, pp. 24-34. 1997.

[15] M. DeMers. "GIS Modeling in Raster". John Wiley and Son, p. 208. New York, USA. 2001.

[16] H. Samet. "Applications of spatial data structures: Computer graphics, image processing, and GIS”. Addison-Wesley Longman Publishing Co. Inc, p. 507. Boston. 1990. 